\title{
Anticancer effects of Poncirus fructus on hepatocellular carcinoma through regulation of apoptosis, migration, and invasion
}

\author{
SUVESH MUNAKARMI $^{1 *}$, LOKENDRA CHAND ${ }^{1 *}$, HYUN BEAK SHIN $^{1,2}$, USAMA KHAMIS HUSSEIN $^{3,4}$, \\ BONG-SIK YUN ${ }^{5}$, HAE RYONG PARK ${ }^{6}$ and YEON JUN JEONG ${ }^{1,2}$
}

\author{
${ }^{1}$ Laboratory of Liver Regeneration, Biomedical Research Institute, Chonbuk National University Medical School; \\ ${ }^{2}$ Department of Surgery, Chonbuk National University Medical School, Jeonju 54907; ${ }^{3}$ Research Institute of Clinical \\ Medicine of Jeonbuk National University, Biomedical Research Institute of Chonbuk National University, Jeonju 54907, \\ Republic of Korea; ${ }^{4}$ Faculty of Science, Beni-Suef University, Beni-Suef 62511, Egypt; ${ }^{5}$ Division of Biotechnology, \\ College of Environmental and BioSource Sciences, Chonbuk National University, Jeonju 54907; ${ }^{6}$ Department of Food Science \\ and Biotechnology, Kyungnam University, Masanhappo-gu, Changwon-si 631-701, Republic of Korea
}

Received May 15, 2020; Accepted September 4, 2020

DOI: 10.3892/or.2020.7790

\begin{abstract}
Poncirus fructus (PF) is a phytochemical compound extracted from the dry, immature fruits of Poncirus trifoliate. $\mathrm{PF}$ is traditionally used to treat gastrointestinal disorders, allergies, and inflammatory disease. In East Asia, PF is also known for its anticancer properties. There are numerous reports on the anticancer and anti-inflammatory effects of PF in a wide range of cancers and gastrointestinal diseases, respectively. However, the role of PF in inducing apoptosis and suppressing the invasiveness of hepatocellular carcinoma (HCC) remains unclear. This study investigated the ability of PF to induce apoptosis and inhibit the invasiveness and migratory ability of HCC cell lines (Hep3B and Huh7). Wound healing, Transwell migration and invasion, and colony-formation assays, as well as flow cytometry, were used to analyze cell proliferation, migration, invasion, and apoptosis. Epithelial-mesenchymal transition (EMT)-related and apoptotic proteins were assessed by western blotting. The mitochondrial membrane potential of the Hep3B and Huh7 cells was observed with tetramethylrhodamine ethyl ester. The reactive oxygen species (ROS) level was determined by dihydroethidium (DHE) staining. PF treatment significantly decreased the proliferation of Hep3B and Huh7 cells in a dose-dependent manner, reduced the mitochondrial membrane potential, increased ROS levels, decreased the protein levels of $\mathrm{Bcl}-2$, and increased the protein levels of
\end{abstract}

Correspondence to: Professor Yeon Jun Jeong, Department of Surgery, Chonbuk National University Medical School, 634-18 Geumam-dong, Duckjin-gu, Jeonju 54907, Republic of Korea E-mail: surgeon@jbnu.ac.kr

${ }^{*}$ Contributed equally

Key words: Poncirus fructus, hepatocellular carcinoma, epithelial to mesenchymal transition, apoptosis, cell invasion, cell migration
Bax and cleaved caspase- 3 and 9, suggesting that PF mediated HCC apoptosis via a mitochondrial pathway. Our findings showed that PF prevented $\mathrm{HCC}$ cell migration and invasion by inhibiting the EMT process and downregulating MMP-2 and MMP-9 activities. The results suggest the potential anticancer effects of PF by inhibiting proliferation, inducing apoptosis, and reducing the invasion and migration of HCC cells.

\section{Introduction}

Hepatocellular carcinoma (HCC) is the fifth most common malignant disease and the third most common cause of cancer-related death worldwide $(1,2)$. A steady increase in viral hepatitis, metabolic syndrome, and alcohol consumption over the past decades has contributed to the increasing incidence of HCC (3). The main cause of treatment failure and death in hepatic cancer patients is the highly metastatic nature and recurrence rate of the disease $(4,5)$. Despite specific guidance for monitoring the disease presented in a variety of guidelines, more than two-thirds of HCC patients are diagnosed at an advanced stage of the disease with limited curative treatment options $(6,7)$. Thus, HCC continues to be a global healthcare problem. Although significant efforts have been made to develop and evaluate chemical compounds for the treatment of HCC, only a limited number of drugs, such as first-line drugs sorafenib and lenvatinib, and second-line drugs regorafenib and cabozantinib have shown success in phase III clinical trials (8-10).

Pin1 is a peptidyl-prolyl cis/trans isomerase, member of the parvulin family of PPIase enzymes, which plays important roles in cell signaling, including conformational changes in protein kinase and cellular substrates. Several studies have suggested that Pin1 plays a significant role in various cellular activities, including apoptosis, cell cycle progression, differentiation, proliferation, and transformation (11-13). Pin1 is frequently overexpressed in many types of cancer including HCC and there is a strong relationship between the expression of Pin1 and $\beta$-catenin/cyclin D1 $(14,15)$. The isomerization 
of Pin1 is important for the stabilization of $\beta$-catenin and cyclin D1 $(15,16)$. Cancer metastasis is a complex step-by-step mechanism involving the detachment of cells from the primary tumor, destruction of the extracellular matrix (ECM), infiltration into the circulatory and lymphatic systems, invasion across the basement membranes into new tissue, and growth (17-19). Past studies have shown that epithelial mesenchymal transition (EMT) plays a significant role in the spread of malignant tumors by rendering tumor cells more susceptible and facilitating the invasion and metastasis of cancer cells (20). Matrix metalloproteinases (MMP-2 and MMP-9) are key enzymes responsible for tumor cell migration, invasion, and metastasis $(21,22)$. Therefore, the suppression of tumor cell EMT and the activation of apoptosis have the potential to treat tumors.

Mitochondria are crucial in controlling cell apoptosis by releasing caspase activators and caspase-independent death effectors, which results in the loss of important mitochondrial function $(23,24)$. Mitochondrial dysfunction contributes to the degradation of mitochondrial membrane potential and, the instability of electron transport reactions, resulting in the overproduction of reactive oxygen species (ROS), caspase activation, and apoptosis pathway initiation (25). Numerous studies have identified bioactive compounds and phytochemical sources with anticancer effects that inhibit cancer cell proliferation and modulate metabolism (26). Our findings suggest that $\mathrm{PF}$ decreased the mitochondrial membrane potential and increased the production of mitochondrial ROS and apoptotic cell death in hepatocellular cancer cells.

Several studies have evaluated the potential pharmacological activity, including the antimicrobial, antifungal, anti-inflammatory, antioxidant, and anticancer effects, of plant-derived compounds (27-30). The current study used Poncirus fructus (PF), a phytochemical extract obtained from the dry immature fruits of Poncirus trifoliata. Poncirus trifoliata grows naturally on Gaduk and Jeju islands in South Korea. It is cultivated by farmers in the southern villages of South Korea. PF is believed to possess anti-inflammatory, anti-allergic, and lipid-lowering properties. PF has traditionally been used to treat gastrointestinal disorders, womb contraction, dyspepsia, and blood circulation-related disorders in East Asia, including South Korea (31-33). The biological activities of $P$. trifoliata, such as oncogenic-attenuating activity, anaphylaxis inhibition, and Helicobacter pylori suppression have been investigated (34). Furthermore, it has been reported that $P$. trifoliata extract is cytotoxic to promyelocytic leukemia cells (35). However, neither the cytotoxic effects of PF on HCC nor the molecular mechanism underlying its oncogenic activity has been elucidated. Therefore, the present study was conducted to investigate the molecular mechanism of PF in proliferation, apoptosis, and tumor metastasis. The findings of this study suggest the potential therapeutic effects of PF to efficiently managing $\mathrm{HCC}$.

\section{Materials and methods}

PF extract preparation. The PF extract used was from the same batch as that in a previous study (36). Briefly, the dry immature fruits of $P$. trifoliata were purchased from an Oriental medical store in Geuman, Korea. The fruits were processed with methanol at room temperature for $24 \mathrm{~h}$. The $\mathrm{PF}$ methanol extract was concentrated under reduced pressure using a rotary evaporator. The residues were resuspended in distilled water and further extracted with hexane and ethyl acetate. The ethyl acetate-soluble fraction was subjected to silica gel chromatography using the phase solvent method and a chloroform-methanol solvent. The active fractions were combined, concentrated, and subjected to chromatography on a Sephadex LH-20 column eluted with methanol. Again, the active fraction was subjected to $\mathrm{C} 18$ reverse-phase column chromatography eluted with $80 \%$ aqueous methanol followed by high-performance liquid chromatography (HPLC) eluted with $85 \%$ aqueous methanol to obtain the desired compound.

Cell culture and drug treatments. Hep3B and Huh7 cells were obtained from the American Type Culture Collection (ATCC). Cell line authentication was systematically conducted using a panel of ATCC short tandem repeats (STR) and routinely monitored for mycoplasma contamination and tested negative for mycoplasma contamination. The cell lines were cultured in Dulbecco's Modified Eagle's Medium (DMEM; HyClone ${ }^{\circledR}$, Thermo Fisher Scientific, Inc.) supplemented with $10 \%$ fetal bovine serum (FBS; HyClone ${ }^{\circledR}$, Thermo Fisher Scientific, Inc.) and $1 \%$ antibiotics (HyClone ${ }^{\circledR}$, Thermo Fisher Scientific, Inc.) at $37^{\circ} \mathrm{C}$ in humidified conditions with $5 \% \mathrm{CO}_{2}$. The cells were treated for $24 \mathrm{~h}$ with $\mathrm{PF}$ dissolved in phosphate-buffered saline (PBS) $(0,20,30$, or $40 \mu \mathrm{M})$.

Cell proliferation assay. Hep3B and Huh7 cells were seeded into 96-well plates at a cell density of $5 \times 10^{3}$ per well and cultured for $24 \mathrm{~h}$. The cells were treated with the indicated drug concentrations $(0,20,30,40 \mu \mathrm{M})$. The medium was removed, and the cells were incubated for $2 \mathrm{~h}$ in solution with $50 \mu \mathrm{l}$ of $5 \mathrm{mg} / \mathrm{ml} \mathrm{2,5-diphenyl} \mathrm{tetrazolium} \mathrm{bromide}$ (MTT; Sigma-Aldrich; Merck KGaA). The transformed purple formazan crystals were solubilized in dimethyl sulfoxide (DMSO) and the optical density was measured at a wavelength of $575 \mathrm{~nm}$.

Colony forming assay. Hep3B and Huh7 cells were seeded in 6 -well plates (1,000 cells per well) for $24 \mathrm{~h}$. The medium was replaced with fresh medium containing PF $(20,30$, or $40 \mu \mathrm{M})$ for $24 \mathrm{~h}$. After $24 \mathrm{~h}$, the medium was removed, and fresh medium was added to the cells for 10-14 days. Afterward, the cells were fixed for $15 \mathrm{~min}$ with ice-cold methanol, washed with PBS, stained with $1 \%$ crystal violet solution (bioWORLD) for $1 \mathrm{~h}$ at room temperature, and the colonies with $>10$ cells were counted by using densitometric software Clono-Counter as described previously (37).

Annexin assay. Apoptosis of the Hep3B and Huh7 cells was detected by flow cytometry. Briefly, Hep3B and Huh7 cells were seeded at a cell density of $5 \times 10^{4}$ in a $60-\mathrm{mm}$ culture dish overnight and treated with medium containing PF $(0,20$, 30 , and $40 \mu \mathrm{M}$ ) for $24 \mathrm{~h}$. The cells were trypsinized and the supernatants containing the cells were collected. The cells were centrifuged at $1,500 \mathrm{rpm}$ for $3 \mathrm{~min}$ at $15^{\circ} \mathrm{C}$ and frequently washed with PBS. Then, an Annexin V-FITC apoptosis kit was used to determine apoptosis by flow cytometry according to the manufacturer's instructions. 
Mitochondrial membrane potential $(\Delta \Psi m)$ analysis. The mitochondrial membrane potential was determined using a tetramethylrhodamine ethyl ester perchlorate (TMRE) mitochondrial membrane potential assay kit (Abcam) according to the manufacturer's instructions. Hep3B and Huh7 cells were seeded at a density of 10,000/well in 96-well plates. The cells were cultured with PF $(0,20,30$, and $40 \mu \mathrm{M})$ for $24 \mathrm{~h}$. Then, the cells were stained with TMRE (400 nmol/l) for $20 \mathrm{~min}$ and washed twice with PBS. The TMRE dye intensity was measured at excitation and emission wavelengths of $549 / 575 \mathrm{~nm}$, respectively, using a fluorescence plate reader.

Reactive oxygen species (ROS) analysis. The levels of intracellular ROS in the Hep3B and Huh7 cells were measured using a fluorescent dihydroethidium (DHE) probe. Intracellular DHE is oxidized to ethidium, which binds to DNA and stains the nuclei a bright red fluorescent color. Hep3B and Huh7 cells were cultured in glass-bottom confocal dishes and pretreated with PF as previously described. The cells were washed twice with PBS and then fixed at room temperature with ice-cold methanol for 10-15 min. Then, DHE was diluted in PBS to a final concentration of $5 \mu \mathrm{M}$ and applied to the cells for $35 \mathrm{~min}$ at $37^{\circ} \mathrm{C}$ in the dark. The cells were labeled with 4',6-diamidino-2-phenylindole, dihydrochloride (DAPI) for 5 min after washing twice with PBS, and examined under a fluorescence microscope using a x63 oil immersion objective lens (Axioskop 2 Plus, Carls Zeiss, Gottingen, Germany).

In vitro cell migration and invasion assays. The migration and invasion potential of the Hep3B and Huh7 cells was evaluated using a Transwell chamber with $8.0-\mu \mathrm{m}$ pore polyester membrane inserts and $8.0-\mu \mathrm{m}$ pore polyester membrane inserts with Matrigel-coated chambers (Corning, Inc.). For the migration assay, Hep3B and Huh7 cells treated with PF at the indicated concentrations were seeded in the upper chamber at a density of $5 \times 10^{4}$ cells per well in $200 \mu \mathrm{l}$ of serum-free medium, and $500 \mu \mathrm{l}$ of $10 \%$ FBS-containing medium was added to the lower chamber. The suspension was discarded after $24 \mathrm{~h}$ and the cells on the top layer of the membrane were removed with cotton swabs. The cells that migrated to the lower membrane surface were stained with the Diff-Quick kit solution. The migrated cells were counted under light microscopy in three randomly chosen fields. The Matrigel-coated chamber was rehydrated with serum-free medium in a humidified tissue culture incubator to perform the invasion assay, followed by the migration assay. After $24 \mathrm{~h}$, the suspension medium was discarded and the cells on the upper layer of the membrane were removed using cotton swabs. The cells that invaded the lower surface of the membrane were fixed with ice-cold methanol and stained with the Diff-Quick kit solution. The invasive cells were detected, photographed, and counted in three randomly chosen areas under light microscopy.

Immunoblot analysis. The cells were washed with PBS and lysed with lysis buffer (iNtRON Biotechnology) containing a cocktail of phosphatase inhibitor-1. Western blotting was performed as previously described (38). The membranes were incubated overnight with primary antibodies specific for anti-rabbit cleaved caspase-3 (cat. no. 9661) and -9, anti-mouse caspase-9 (cat. no. 9508), cleaved PARP (cat. no. 5625), E-cadherin (cat. no. 3195), vimentin (cat. no. 5741), Snail (cat. no. 3879), MMP-9 (cat. no. 2270) (dilution 1:1,000; Cell Signaling Technology, Inc.), anti-mouse actin, $\alpha$-SMA (cat. no. A2547, dilution 1:1,000; Sigma-Aldrich; Merck KGaA), anti-rabbit Bcl-2 (cat. no. BS1511), Bax (cat. no. BS6420), MMP-2 (cat.no.BS1236) (dilution 1:1,000; Bioworld Tech.Inc.), anti-rabbit caspase-3 (cat. no. sc-7148), anti-mouse Pin1 (cat. no. sc-46660), anti-rabbit cyclin D1 (cat. no. sc-717) (dilution 1:1,000, Santa Cruz Biotechnology, Inc.), anti-mouse $\beta$-catenin (cat. no. 610154), and N-cadherin (cat. no. 610920) (dilution 1:1,000, BD Biosciences). The membranes were washed and incubated with horseradish peroxidase (HRP)-conjugated anti-mouse (cat. no. ADI-SAB-100-J) or anti-rabbit secondary antibodies (ADI-SAB-300-J) (dilution 1:3,000, Enzo Life Sciences). Protein expression was detected using an enhanced chemiluminescence detection kit (Millipore Corp.).

Gelatin zymography. Gelatin zymography was performed to determine the levels of MMPs, such as MMP-2 and MMP-9. The HCC cell supernatants were collected by centrifugation at $4,000 \mathrm{x} \mathrm{g}$ at $4^{\circ} \mathrm{C}$ and the protein content was determined using a BCA protein assay kit. Equal amounts of protein mixed with non-reducing $6 \mathrm{X}$ loading buffer were separated by SDS-PAGE containing $0.1 \%$ gelatin. After electrophoresis, the gels were rinsed thrice with $0.25 \%$ Triton $\mathrm{X}-100$ for $15 \mathrm{~min}$ at room temperature and then incubated at $37^{\circ} \mathrm{C}$ in developing buffer $\left(50 \mathrm{mM}\right.$ Tris-HCL pH 7.4, $5 \mathrm{mM} \mathrm{CaCl}_{2}$, $200 \mathrm{mM} \mathrm{NaCl}$ ) for $42 \mathrm{~h}$. After incubation, the MMP-2 and MMP-9 activities were measured by Coomassie blue staining and the gel was imaged using an LAS 3000 (Fuji) (39).

Statistical analysis. All the experimental results were obtained by repeating each experiment at least three times and data are expressed as means \pm standard deviation. Statistical significance was analyzed by one-way analysis of variance (ANOVA) using the Dunnett's test or Turkey's post hoc test in Prism 7 software (GraphPad Software, Inc.). A $\mathrm{P}$-value less than $0.05(\mathrm{P}<0.05)$ was considered statistically significant.

\section{Results}

Anti-proliferative effects of PF in the cancer cells. MTT and colony-forming assays were performed to evaluate the anti-proliferative effects of PF in Hep3B and Huh7 cells. As shown in Fig. 1A, PF significantly reduced the cell proliferation of Hep3B and Huh7 cells in a dose-dependent manner. The inhibitory effect of PF on cell proliferation was also measured using a clonogenic assay. As shown in Fig. 1B and C, $\mathrm{PF}$ significantly inhibited the colony formation of Hep3B and Huh7 cells in a dose-dependent manner. These results indicate that PF had a significant inhibitory impact on the viability and proliferation of HCC cell lines.

PF promotes the apoptosis of the HCC cells. To explore whether PF induced cancer cell apoptosis, flow cytometry was conducted. As shown in Fig. 2A and B, treatment with PF significantly induced apoptosis in the Hep3B and Huh7 cells in a dose-dependent manner compared to the control. 

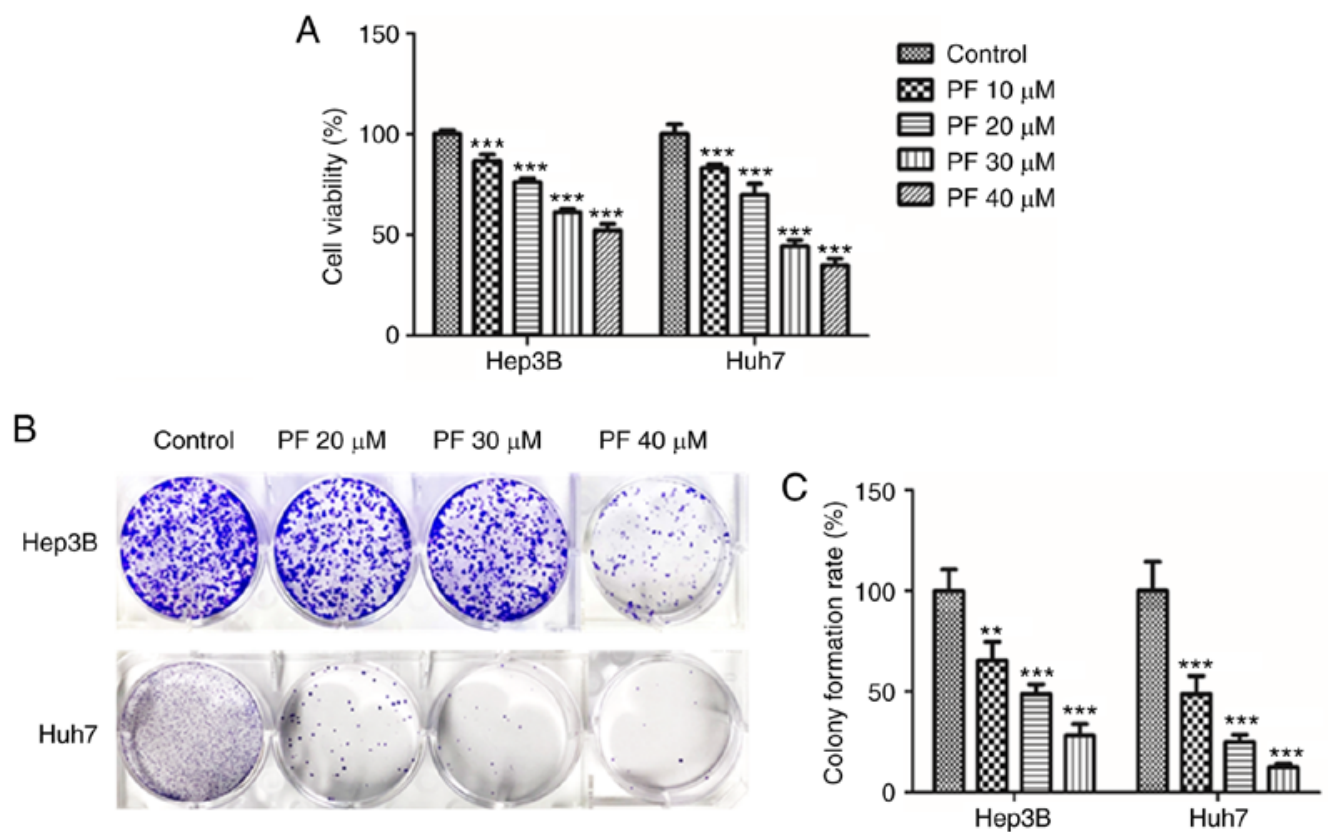

Figure 1. PF inhibits the proliferation of Hep3B and Huh7 cells. (A) PF (10-40 $\mu \mathrm{M})$ inhibited the proliferation of cells in HCC Hep3B and Huh7 as determined by the MTT assay. (B) PF (20-40 $\mu \mathrm{M})$ inhibited the ability of Hep3B and Huh7 cell colony formation. (C) Quantitative analysis of the number of colonies in PF-treated cells compared with that of the control group. Surviving colonies with $>10$ cells were counted. The data represents the results from three independent experiment and expressed as mean $\pm \mathrm{SE} .{ }^{* *} \mathrm{P}<0.01,{ }^{* * *} \mathrm{P}<0.001$, significant difference with respect to the control group. PF, Poncirus fructus; HCC, hepatocellular carcinoma.

PF promotes apoptosis of HCC cells by reducing mitochondrial membrane potential $(\Delta \Psi m)$ and increasing $R O S$. The present study demonstrated the mechanism of PF-induced apoptosis on HCC cells (Hep3B and Huh7) by analyzing the apoptosis-related proteins, and quantifying the mitochondrial membrane potential $(\Delta \Psi \mathrm{m})$ and intracellular ROS levels. As shown in Fig. 3A, western blot analysis revealed that PF significantly decreased the expression of procaspase-3, procaspase-9, PARP and anti-apoptotic protein $\mathrm{Bcl} 2$, whereas it increased the expression of apoptotic proteins Bax, cleaved caspase-3, cleaved caspase- 9 and cleaved PARP in a dose-dependent manner. The loss of mitochondrial membrane potential $(\Delta \Psi \mathrm{m})$ results in mitochondrial membrane permeabilization, which is regarded as an important hallmark of early apoptosis and plays a key role in the intrinsic apoptotic pathway (40-42). Therefore, we further analyzed the disruption of the mitochondrial membrane by PF. As shown in Fig. 3B, PF induced a significant, concentration-dependent depletion of mitochondrial membrane potential. Mitochondria are considered the main source of ROS, which is considered a trigger of apoptosis (43). Therefore, we determined the intracellular ROS generation in Hep3B and Huh7 cells by assessing the DHE fluorescence intensity after PF treatment. As shown in Fig. 3C, PF treatment induced stronger fluorescence intensity in Hep3B and Huh7 cells compared to the control, which indicated increased levels of ROS. These results suggest that PF induced apoptosis of the Hep3B and Huh7 cells by effects on the mitochondrial apoptosis pathway.

PF inhibits the migration and invasion of HCC cells. The migratory and invasive abilities of tumor cells are considered as significant hallmarks of HCC and play a key roles in the metastasis of various tumors (44). To investigate whether PF exhibits anti-migratory and anti-invasion effects, Transwell assays were performed. As shown in Fig. 4A and B, the migratory ability of Hep3B and Huh7 cells was inhibited by PF treatment in a dose-dependent manner. Consistently, the Transwell invasion assay showed that $\mathrm{PF}$ treatment inhibited the number of invasive cells (Fig. 4C and D).

Effects of PF on the expression of Pin 1, cyclin D1, $\beta$-catenin l, and EMT marker. EMT is vital in tumor cell growth, causing the invasion and metastasis of malignant tumors (45). Therefore, the protein levels of Pin 1, cyclin D1, and EMT markers such as $\beta$-catenin, E-cadherin, vimentin, $\mathrm{N}$-cadherin, Snail, and $\alpha$-SMA hepatic cancer cells were evaluated. As shown in Fig. 5A, the treatment of Hep3B and Huh7 cells with PF markedly increased the expression of E-cadherin, whereas it decreased the levels of Pin1, cyclin D1, $\beta$-catenin, $N$-cadherin, vimentin, Snail, and $\alpha$-SMA in a dose-dependent manner. These results suggested that PF inhibited cancer cell progression by decreasing the EMT process. A previous study demonstrated that matrix metalloproteinases (MMPs) such as MMP-2 and MMP-9 play crucial roles in basement membrane degradation during tumor cell invasion (46). Therefore, we determined the expression of MMP-2 and MMP-9 in Hep3B and Huh7 cells. As shown in Fig. 5B, PF treatment markedly downregulated the protein levels of MMP-2 and MMP-9. To confirm whether the activities of extracellular MMPs were inhibited by PF, a gelatin zymography assay was performed. As shown in Fig. 5C, PF decreased the activities of MMP-2 and MMP-9 in the Huh7 culture medium. These results suggest that PF inhibits cancer cell migration and invasion by attenuating the activities of the metalloproteinases. 

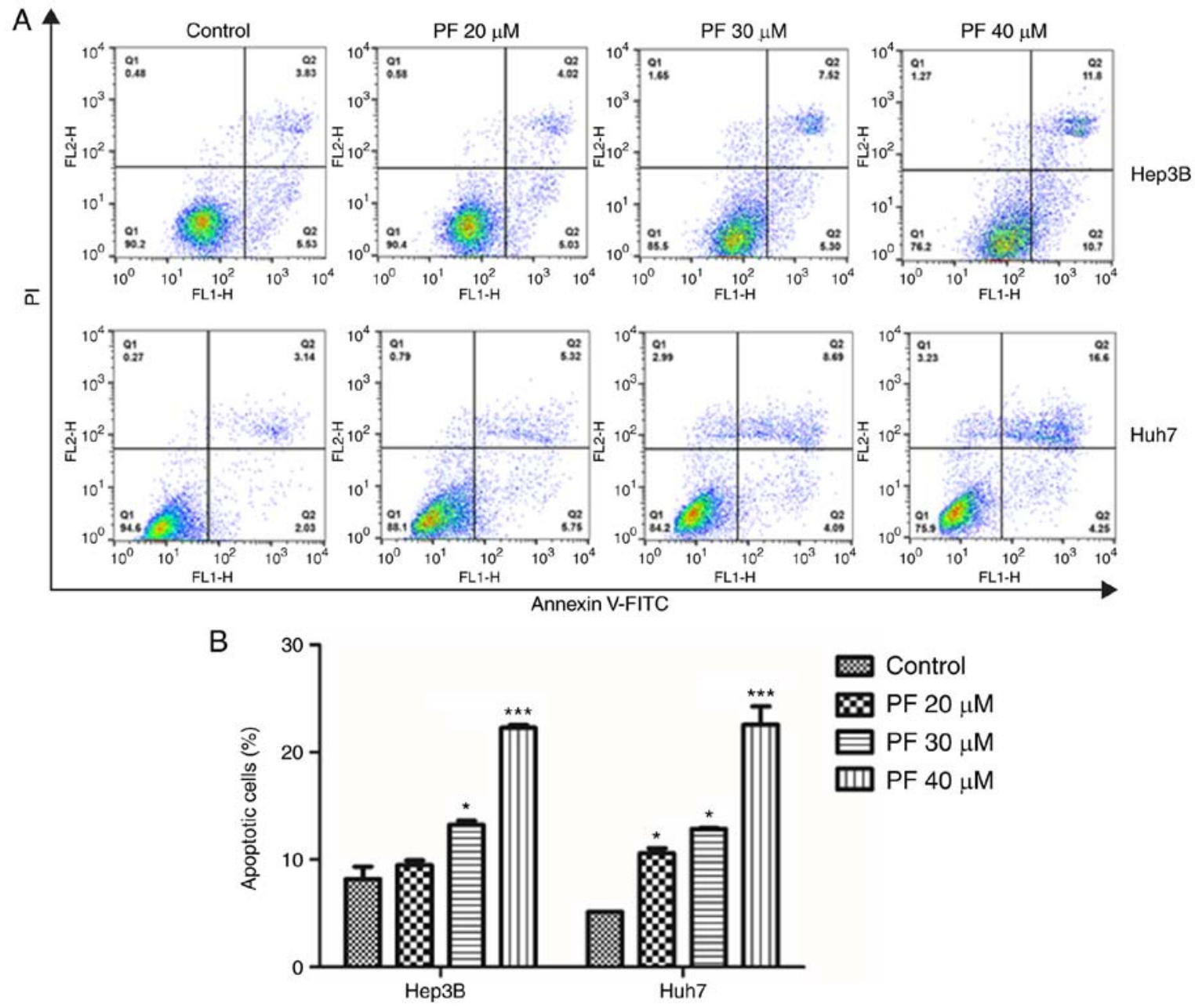

Figure 2. PF induces HCC cell apoptosis. (A) Annexin V/propidium iodide (PI) staining of Hep3B and Huh7 cells after treatment with PF (20, 30 and $40 \mu \mathrm{M}$ ), as determined by fluorescence-activated cell sorting (FACS) analysis. (B) Quantitative assessment (\%) of the apoptotic cells. The data represents the results from three independent experiment and expressed as mean $\pm \mathrm{SE}$. ${ }^{*} \mathrm{P}<0.05,{ }^{* * * *} \mathrm{P}<0.001$, significant variation relative to the control group. $\mathrm{PF}$, Poncirus fructus; HCC, hepatocellular carcinoma.

\section{Discussion}

The advancements, discoveries, and successful trials of plant-derived drugs have directed attention to treating various cancers with agents with low toxicity to normal cells. Poncirus fructus (PF) is a phytochemical compound extracted from the dry, immature fruits of Poncirus trifoliate. $\mathrm{PF}$ is traditionally used for the treatment of gastrointestinal and vascular disorders in South Asia. Previous studies have reported the use of PF for the clinical treatment of various cancers (47). However, no strong evidence has been provided on the molecular mechanism of the anticancer role of PF (35). Several previous studies have shown that the pharmacological efficacy of these fruits depends on their maturity. The previous studies on PF in cancer and non-cancer diseases are documented in Table SI. Hepatocellular carcinoma (HCC) is the most common malignancy in many countries, with a low survival rate and a high rate of intrahepatic and extra-hepatic metastasis $(48,49)$. Hepatic cancer patients have high recurrence and mortality rates due to progression by tumor cell migration and invasion (5). The present study showed the antiproliferative and anti-invasive effects of $\mathrm{PF}$ by inducing apoptosis in HCC Hep3B and Huh7 HCC cell lines. These results suggest the potential of PF as a clinical agent for cancer treatment.

We demonstrated that the high expression of Pin1, peptidyl-prolyl cis/trans isomerase, in HCC was reduced by treatment with PF. The dysregulation of PIN1 contributes to pathological conditions including cancer (50). A number of cancer markers such as cyclin D1, $\beta$-catenin, and NF- $\mathrm{kB} / \mathrm{P} 65$ are also correlated with the overexpression of PIN1 (15,51-54). Several studies have indicated that Pin $1, \beta$-catenin, and cyclin D1 were involved in promoting the growth of HCC by regulating cell proliferation and reducing the activity of E-cadherin-mediated cell adhesion and thus, were correlated with poorly differentiated disease in HCC patients (55-59). In the present study, we found that PF inhibited epithelial mesenchymal transition (EMT) by reducing the expression of Pin 1, $\beta$-catenin, and cyclin D1. EMT plays a significant role in the malignancy of tumors by reducing the ability of cells to bind to the basement membranes, inducing a loss of epithelial cell polarity, and acquiring elongated mesenchymal morphology and migratory and invasive properties to become mesenchymal stem cells (60-62). Transwell migration and invasion assays are commonly used to evaluate EMT. During EMT, epithelial cells acquire motile and invasive characteristics by increasing 

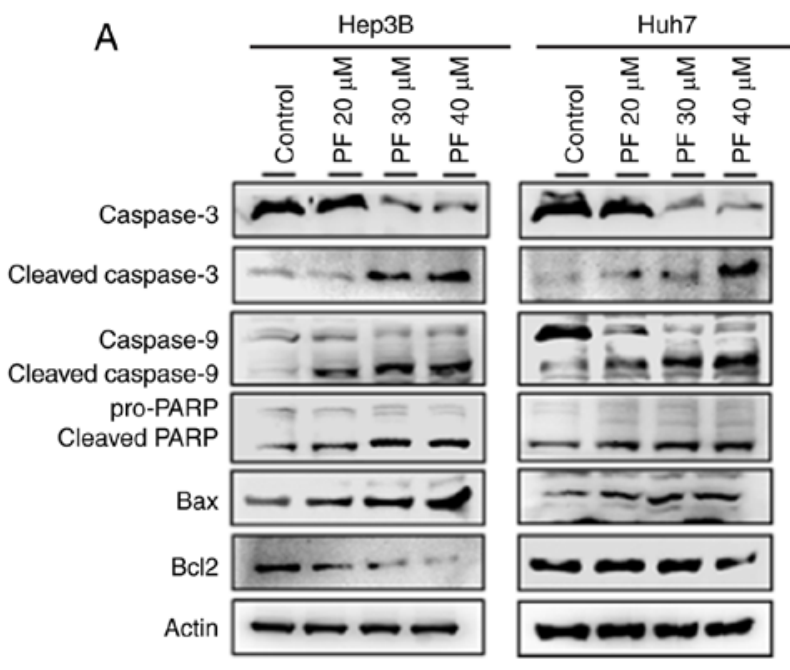

B

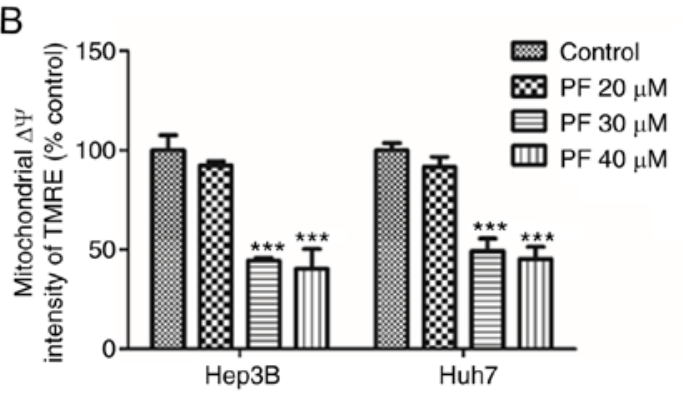

C
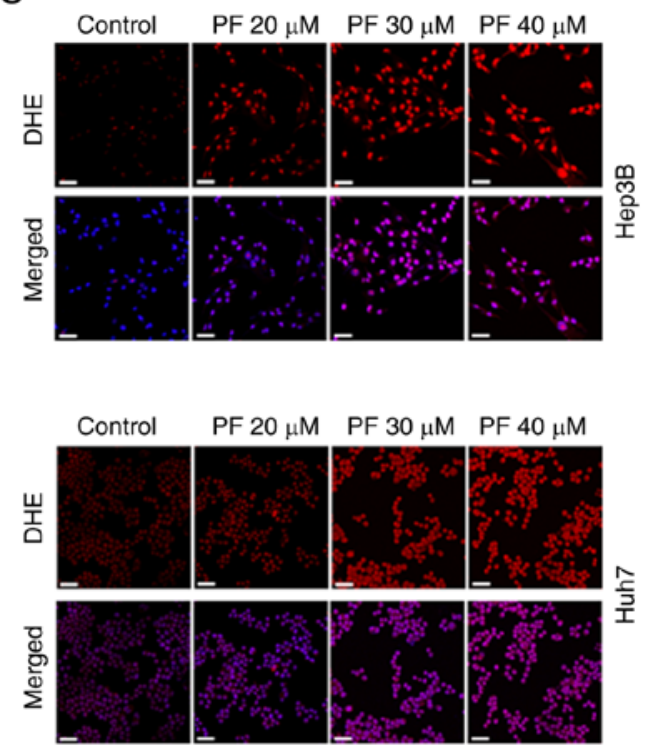

Figure 3. PF induces HCC cell apoptosis by increasing ROS and disrupting mitochondrial membrane potential. (A) Western blot analysis was utilized to determine the levels of apoptosis-related proteins. (B) PF-induced changes in the mitochondrial membrane potential $(\Delta \Psi \mathrm{m})$ of Hep3B and Huh7 cells were determined with an ELISA reader after tetramethylrhodamine ethyl ester perchlorate (TMRE) staining. (C) The intracellular ROS levels in Hep3B and Huh7 cells after PF treatment. The cells were incubated with the ROS fluorescent probe, dihydroethidium (DHE), and evaluated using a confocal microscope. Scale bar, $30 \mu \mathrm{m}$. The data represents the results from three independent experiment and expressed as mean $\pm \mathrm{SE} .{ }^{* * *} \mathrm{P}<0.001$, significant difference with respect to the control group. PF, Poncirus fructus; HCC, hepatocellular carcinoma; ROS, reactive oxygen species.
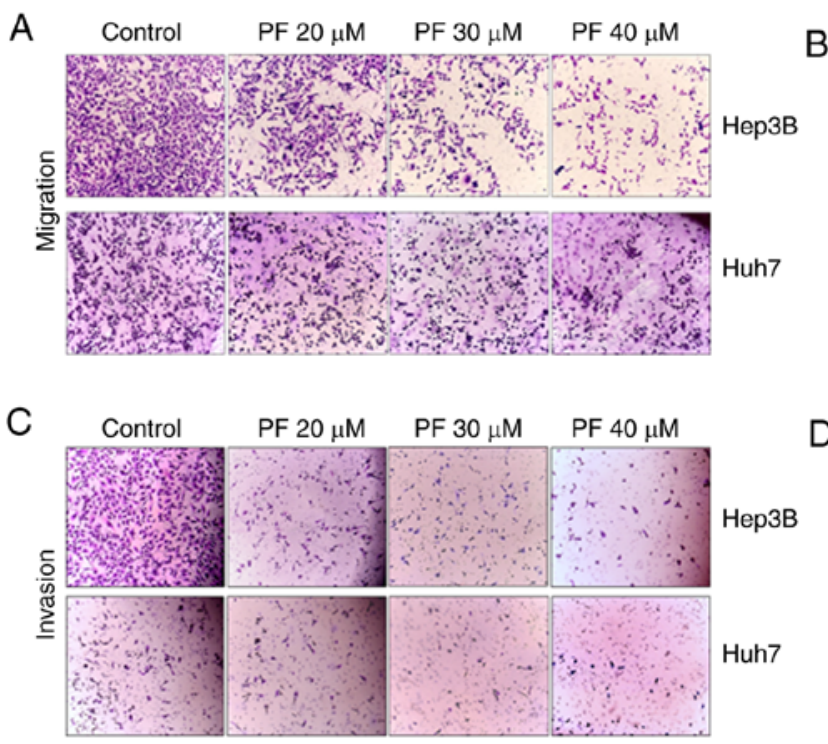

B
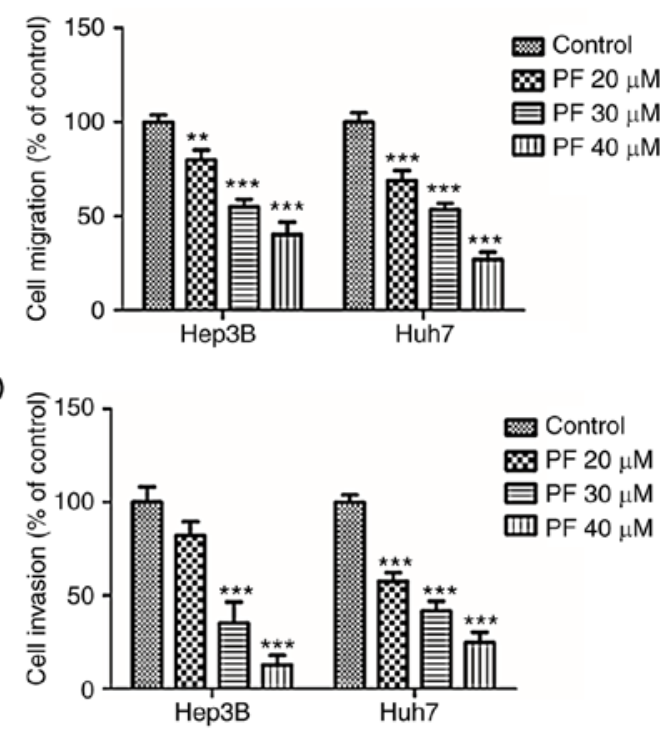

Figure 4. PF inhibits HCC cell migration and invasion. (A and C) The effect of PF (20,30 and $40 \mu \mathrm{M})$ on the migration and invasion abilities of Hep3B and Huh7 cells was examined by Transwell assays. (B and D) Quantification of the migration and invasion abilities of the Hep3B and Huh7 cells compared to the control. The chambers were stained with diff-quik stain kit, and the number of migrated or invaded cells were counted in five microscopic fields in each well at a magnification of $\mathrm{x} 200 .{ }^{* *} \mathrm{P}<0.01,{ }^{* * *} \mathrm{P}<0.001$, significant difference with regard to the control group. PF, Poncirus fructus; HCC, hepatocellular carcinoma.

mesenchymal properties, including the increased expression of mesenchymal markers $\alpha$-SMA, N-cadherin, and vimentin, and EMT transcriptional factors Slug and Snail, which result in the loss of epithelial markers E-cadherin and ZO-1 (63). 

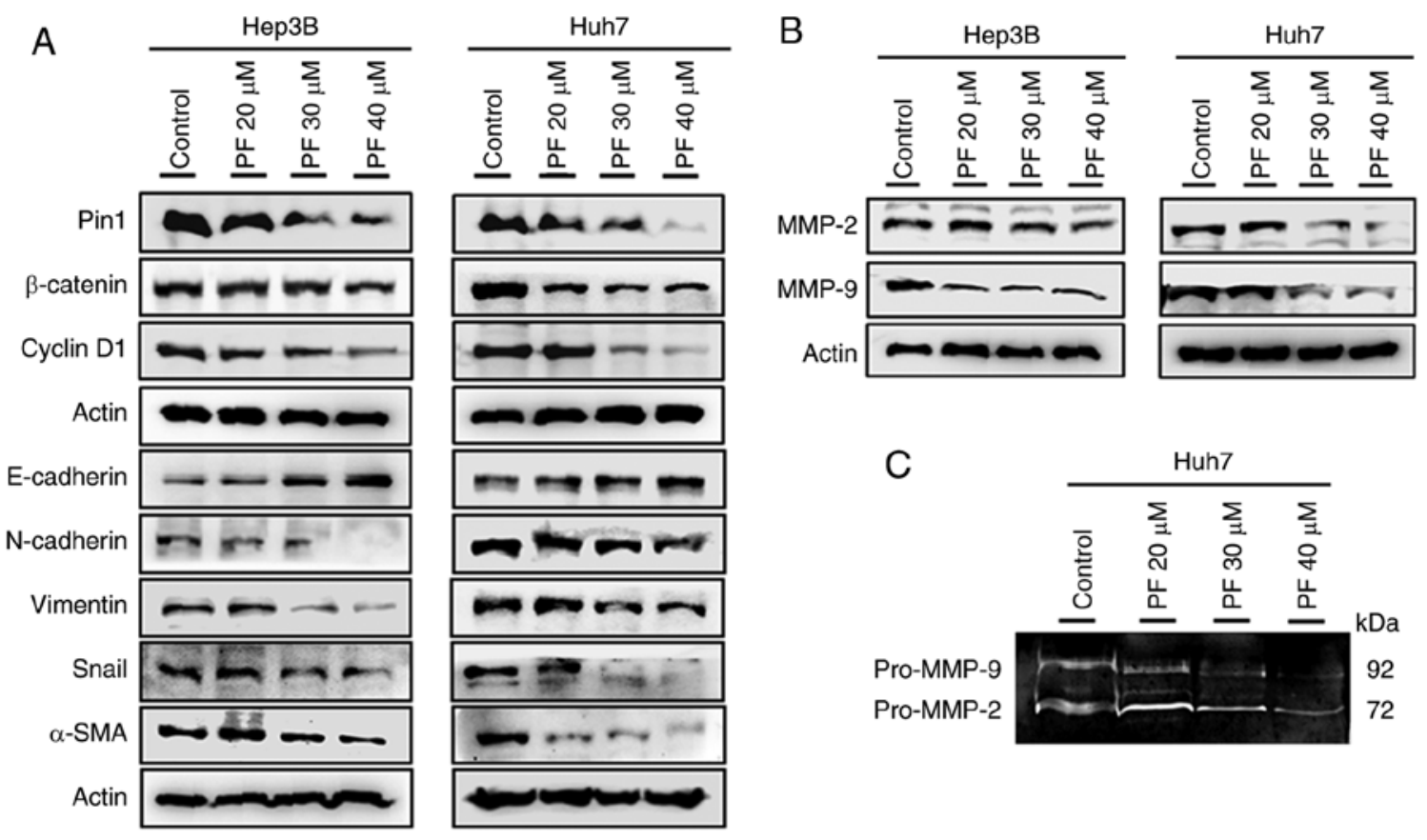

Figure 5. Effects of PF on the expression of Pin1, cyclin D1, $\beta$-catenin and EMT markers in HCC Hep3B and Huh7 cells. (A) Evaluation of Pin1, cyclin D1, $\beta$-catenin and EMT-associated protein expression by western blot analysis. (B) The protein levels of MMP-2 and MMP-9 in Hep3B and Huh7 cells after PF treatment was detected by western blot analysis. (C) The detection of pro-MMP-2 and pro-MMP-9 activity in supernatants was determined by gelatin zymography assay. PF, Poncirus fructus; HCC, hepatocellular carcinoma; MMP, matrix metalloproteinase.

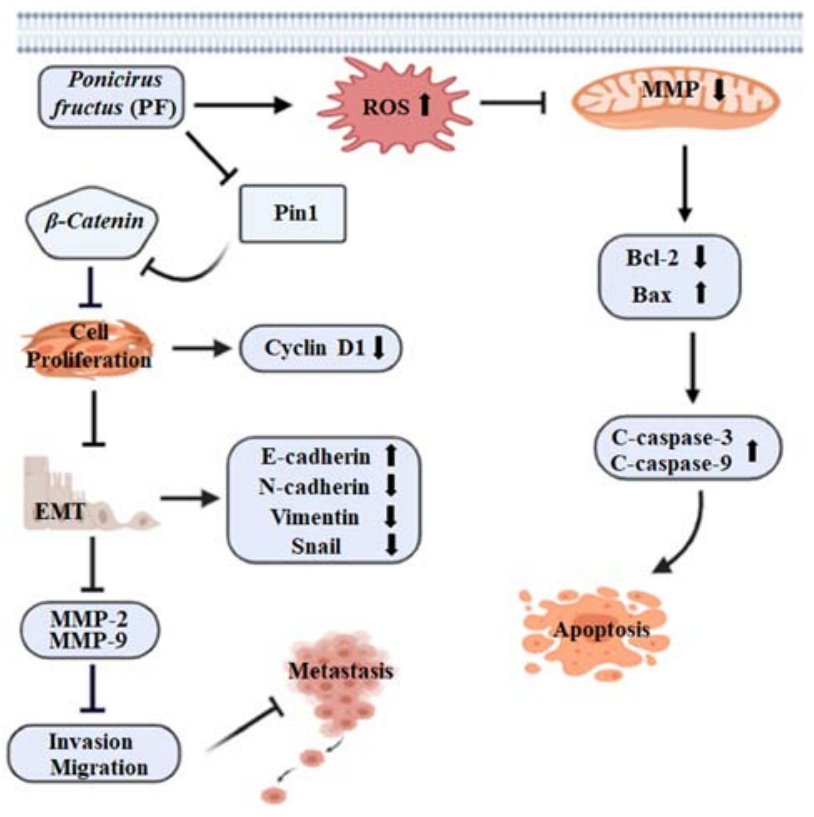

Figure 6. Schematic diagram of the proposed mechanism by which PF exhibits anticancer effects by inducing ROS and disrupting mitochondrial membrane potential, resulting in hepatic cancer cell apoptosis and tumor growth suppression via inhibition of cancer cell invasion and migration ability. ROS, reactive oxygen species; MMP, matrix metalloproteinase; EMT, epithelial mesenchymal transition.

This study, demonstrated that PF inhibited the migration and invasion of hepatic cancer cells. PF treatment also increased the protein levels of E-cadherin and decreased the protein levels of $\alpha$-SMA, $N$-cadherin, and vimentin. The extracellular matrix prevents the metastasis of tumor cells by acting as a physical barrier, and its degradation plays a crucial role in promoting tumor cell migration and invasion (64). MMPs are important proteinases involved in extracellular matrix degradation, and increased MMP expression was shown to promote the invasion of tumor cells $(65,66)$. MMPs such as MMP-2 and MMP-9 are strongly expressed in metastatic tumors and are responsible for the degradation of extracellular matrix basic skeleton IV collagen $(67,68)$. The present study, showed that the expression of MMP-2 and MMP-2 in HCC cells was adequately inhibited by $\mathrm{PF}$ treatment. Together, these findings suggest that $\mathrm{PF}$ reduced HCC cell migration and invasion by reducing the expression of Pin $1, \beta$-catenin, and cyclin D1 thereby inhibiting EMT and downregulating MMP-2 and MMP-9 activity.

Apoptosis plays a key role in the elimination of dysfunctional and damaged cells to maintain homeostasis. Numerous evidence has shown that defects in apoptotic pathways stimulate carcinogenesis and cancer cell survival (69). Therefore, inducing apoptosis in tumor cells may be considered a potential therapeutic approach to treat cancers. There are two main pathways for the initiation of apoptosis, the intrinsic (mitochondria-mediated), and the extrinsic apoptotic pathways (70). Previous studies indicate that proteins of the Bcl-2 member family and caspase cascades perform important regulatory roles in mitochondrial apoptosis (71). The cytosolic localization of Bax pro-apoptotic proteins combines with membrane-bound $\mathrm{Bcl} 2$ to create a heterodimer that stabilizes the cytoplasmic localization of pro-apoptotic proteins and inhibits apoptosis (72). However, the dissociation of Bax from the $\mathrm{Bcl}-2 / \mathrm{Bax}$ heterodimer by the number of the apoptosis-inducing signals allows it to translocate to the mitochondrial membrane to induce apoptosis (73). In this study, we evaluated the effect of PF on apoptosis and apoptosis-related proteins in HCC cell lines. Our data indicate 
that PF significantly increased the levels of pro-apoptotic proteins, such as Bax, cleaved caspase-3, cleaved caspase-9 and cleaved PARP, whereas PF treatment decreased the levels of caspase-3, caspase-9, PARP and anti-apoptotic Bcl-2 protein in a concentration-dependent manner.

Mitochondria are associated with the regulation of cellular apoptosis, tumorigenesis, and drug tolerance. Several studies have reported that the mitochondrial signal transduction pathway has particular importance in the metabolism of cellular energy and the initiation of apoptosis (74). The depletion of mitochondrial membrane potential $(\Delta \Psi \mathrm{m})$ and increased membrane permeability are characteristics of mitochondrial injury. This process releases apoptogenic factors from the mitochondria into the cytosol and reduces oxidative phosphorylation, resulting in mitochondrial-mediated apoptosis (75). Recent research has found that the excessive production of ROS from mitochondria leads to mitochondrial membrane depolarization, which induces apoptosis (76). In the present study, PF treatment reduced mitochondrial membrane potential, which resulted in excessive ROS production in Hep3B and Huh7 cells and induced apoptosis. However, the antitumor effects of PF need to confirmed by future studies using an animal model.

The results of this study revealed that PF effectively inhibited the proliferation and mediated the apoptosis of HCC cells through the mitochondrial apoptotic pathway. In addition, $\mathrm{PF}$ treatment exerted anti-migration and anti-invasive effects on HCC cells by inhibiting EMT and reducing the levels of MMP-2 and MMP-9. The findings that PF induced the apoptosis of Hep3B and Huh7 HCC cells by increasing ROS and disrupting the mitochondrial membrane potential suggest the potential of $\mathrm{PF}$ as a promising $\mathrm{HCC}$ drug candidate. In addition, $\mathrm{PF}$ reduced tumor growth and metastasis by inhibiting cell proliferation and suppressing the migration and invasion ability of Hep3B and Huh7 cells (Fig. 6). These results indicate that Poncirus fructus could be a prospective candidate for treating hepatic cancer.

\section{Acknowledgements}

Not applicable.

\section{Funding}

This research was funded by research grants from the Research Institute of Clinical Medicine of Chonbuk National University and Biomedical Research Institute of Chonbuk National University Hospital.

\section{Availability of data and materials}

The datasets used during the present study are available from the corresponding author upon reasonable request.

\section{Authors' contributions}

SM, LC and YJJ conceived and designed the experiments. UKH performed the zymography experiment. YJJ acted as the chief investigator and provided strategic advice for all aspects of the projects. SM conducted the experiments and interpreted the results with assistance from HBS. B-SY and HRP prepared the materials and agents for the experimental research. SM composed the manuscript. All authors read and accepted the final manuscript for publication.

\section{Ethics approval and consent to participate}

Not applicable.

\section{Patient consent for publication}

Not applicable.

\section{Competing interests}

The authors declare no competing interests.

\section{References}

1. Naghavi M, Wang H, Lozano R, Davis A, Liang X, Zhou M, Vollset SE, Ozgoren AA, Abdalla S, Abd-Allah F, et al: Global, regional, and national age-sex specific all-cause and cause-specific mortality for 240 causes of death, 1990-2013: A systematic analysis for the global burden of disease study 2013. Lancet 385: 117-171, 2015.

2. Mederacke I: Liver fibrosis-mouse models and relevance in human liver diseases. Z Gastroenterol 51: 55-62, 2013.

3. El-Serag HB: Hepatocellular carcinoma. N Engl J Med 365: 1118-1127, 2011.

4. Morise Z, Kawabe N, Tomishige H, Nagata H, Kawase J, Arakawa S, Yoshida R and Isetani M: Recent advances in liver resection for hepatocellular carcinoma. Front Surg 1: 21, 2014.

5. Yu Y, Shen H, Yu H, Zhong F, Zhang Y, Zhang C, Zhao J, $\mathrm{Li} \mathrm{H}$, Chen J, Liu Y and Yang P: Systematic proteomic analysis of human hepotacellular carcinoma cells reveals molecular pathways and networks involved in metastasis. Mol Biosyst 7: 1908-1916, 2011.

6. European Association For The Study Of The Liver; European Organisation For Research And Treatment Of Cancer: EASL-EORTC clinical practice guidelines: Management of hepatocellular carcinoma. J Hepatol 56: 908-943, 2012.

7. Rosen HR, Ghany MG, Chung RT and Lok ASF: NAM 2017 report: A national plan to eliminate hepatitis $B$ and $C$ in the United States by 2030 and the AASLD's response. Hepatology 66: 1020-1022, 2017

8. Llovet JM, Ricci S, Mazzaferro V, Hilgard P, Gane E, Blanc JF, de Oliveira AC, Santoro A, Raoul JL, Forner A, et al: Sorafenib in advanced hepatocellular carcinoma. N Engl J Med 359: 378-390, 2008.

9. Nault JC, Galle PR and Marquardt JU: The role of molecular enrichment on future therapies in hepatocellular carcinoma. J Hepatol 69: 237-247, 2018.

10. Bruix J, Qin S, Merle P, Granito A, Huang YH, Bodoky G, Pracht M, Yokosuka O, Rosmorduc O, Breder V, et al: Regorafenib for patients with hepatocellular carcinoma who progressed on sorafenib treatment (RESORCE): A randomised, double-blind, placebo-controlled, phase 3 trial. Lancet 389: 56-66, 2017.

11. Lu KP, Hanes SD and Hunter T: A human peptidyl-prolyl isomerase essential for regulation of mitosis. Nature 380: 544-547, 1996.

12. Zheng H, You H, Zhou XZ, Murray SA, Uchida T, Wulf G, Gu L, Tang X, Lu KP and Xiao ZX: The prolyl isomerase Pin1 is a regulator of p53 in genotoxic response. Nature 419: 849-853, 2002.

13. Bernis C, Vigneron S, Burgess A, Labbé JC, Fesquet D, Castro A and Lorca T: Pin1 stabilizes Emi1 during G2 phase by preventing its association with SCF(betatrcp). EMBO Rep 8: 91-98, 2007.

14. Liou YC, Ryo A, Huang HK, Lu PJ, Bronson R, Fujimori F, Uchida T, Hunter T and Lu KP: Loss of Pin1 function in the mouse causes phenotypes resembling cyclin D1-null phenotypes. Proc Natl Acad Sci USA 99: 1335-1340, 2002. 
15. Ryo A, Nakamura M, Wulf G, Liou YC and Lu KP: Pin1 regulates turnover and subcellular localization of beta-catenin by inhibiting its interaction with APC. Nat Cell Biol 3: 793-801, 2001.

16. Ryo A, Nakamura M, Wulf G, Liou YC and Lu KP: Pin1 regulates turnover and subcellular localization of beta-catenin by inhibiting its interaction with APC. Nat Cell Biol 3: 793-801, 2001.

17. Chen K, Zhang S, Ji Y, Li J, An P, Ren H, Liang R, Yang J and Li Z: Baicalein inhibits the invasion and metastatic capabilities of hepatocellular carcinoma cells via down-regulation of the ERK pathway. PLoS One 8: e72927, 2013.

18. Gupta GP and Massagué J: Cancer metastasis: Building a framework. Cell 127: 679-695, 2006.

19. Meyer T and Hart IR: Mechanisms of tumour metastasis. Eur J Cancer 34: 214-221, 1998

20. Radisky ES and Radisky DC: Matrix metalloproteinase-induced epithelial-mesenchymal transition in breast cancer. J Mammary Gland Biol Neoplasia 15: 201-212, 2010

21. Song J, Zhang X, Ge Q, Yuan C, Chu L, Liang HF, Liao Z, Liu Q, Zhang $\mathrm{Z}$ and Zhang B: CRISPR/Cas9-mediated knockout of HBsAg inhibits proliferation and tumorigenicity of HBV-positive hepatocellular carcinoma cells. J Cell Biochem 119: 8419-8431, 2018.

22. Itoh $\mathrm{Y}$ and Nagase $\mathrm{H}$ : Matrix metalloproteinases in cancer. Essays Biochem 38: 21-36, 2002.

23. Green DR and Kroemer G: The pathophysiology of mitochondrial cell death. Science 305: 626-629, 2004

24. Yuan H, Mutomba M, Prinz I and Gottlieb RA: Differential processing of cytosolic and mitochondrial caspases. Mitochondrion 1: 61-69, 2001.

25. Zaidieh T, Smith JR, Ball KE and An Q: ROS as a novel indicator to predict anticancer drug efficacy. BMC Cancer 19: 1224 2019.

26. Newman DJ and Cragg GM: Natural products as sources of new drugs from 1981 to 2014. J Nat Prod 79: 629-661, 2016.

27. Vallejo MJ, Salazar L and Grijalva M: Oxidative stress modulation and ROS-mediated toxicity in cancer: A review on in vitro models for plant-derived compounds. Oxid Med Cell Longev 2017: 4586068, 2017

28. Greenwell M and Rahman PK: Medicinal Plants: Their use in anticancer treatment. Int J Pharm Sci Res 6: 4103-4112, 2015.

29. Zheng X, Zhao MG, Jiang CH, Sheng XP, Yang HM, Liu Y, Yao XM, Zhang $J$ and Yin ZQ: Triterpenic acids-enriched fraction from Cyclocarya paliurus attenuates insulin resistance and hepatic steatosis via PI3K/Akt/GSK3 $\beta$ pathway. Phytomedicine 66: 153130, 2020.

30. Sumorek-Wiadro J,Zając A, Maciejczyk A and Jakubowicz-Gil J: Furanocoumarins in anticancer therapy-for and against. Fitoterapia 142: 104492, 2020

31. Yu DJ, Jun JH, Kim TJ, Suh DK, Youn DH and Kim TW: The relaxing effect of Poncirus fructus and its flavonoid content on porcine coronary artery. Lab Anim Res 31: 33-39, 2015.

32. Kornblum HI, Raymon HK, Morrison RS, Cavanaugh KP, Bradshaw RA and Leslie FM: Epidermal growth factor and basic fibroblast growth factor: Effects on an overlapping population of neocortical neurons in vitro. Brain Res 535: 255-263, 1990.

33. Jang Y, Kim EK and Shim WS: Phytotherapeutic effects of the fruits of Poncirus trifoliata (L.) Raf. on cancer, inflammation, and digestive dysfunction. Phytother Res 32: 616-624, 2018

34. Hong J, Min HY, Xu GH, Lee JG, Lee SH, Kim YS, Kang SS and Lee SK: Growth inhibition and G1 cell cycle arrest mediated by 25 -methoxyhispidol A, a novel triterpenoid, isolated from the fruit of Poncirus trifoliata in human hepatocellular carcinoma cells. Planta Med 74: 151-155, 2008.

35. Yi JM, Kim MS, Koo HN, Song BK, Yoo YH and Kim HM: Poncirus trifoliata fruit induces apoptosis in human promyelocytic leukemia cells. Clin Chim Acta 340: 179-185, 2004.

36. Choi AR, Lee IK, Woo EE, Kwon JW, Yun BS and Park HR New glabretal triterpenes from the immature fruits of Poncirus trifoliata and their selective cytotoxicity. Chem Pharm Bull (Tokyo) 63: 1065-1069, 2015

37. Niyazi M, Niyazi I and Belka C: Counting colonies of clonogenic assays by using densitometric software. Radiat Oncol 2: 4, 2007.

38. Munakarmi S, Chand L, Shin HB, Jang KY and Jeong YJ: Indole-3-carbinol derivative DIM mitigates carbon tetrachloride-induced acute liver injury in mice by inhibiting inflammatory response, apoptosis and regulating oxidative stress. Int J Mol Sci 21: $2048,2020$.
39. Ahmed AG, Hussein UK, Ahmed AE, Kim KM, Mahmoud HM, Hammouda O, Jang KY and Bishayee A: Mustard seed (Brassica nigra) extract exhibits antiproliferative effect against human lung cancer cells through differential regulation of apoptosis, cell cycle, migration, and invasion. Molecules 25: 2069, 2020.

40. Skulachev VP: Why are mitochondria involved in apoptosis? Permeability transition pores and apoptosis as selective mechanisms to eliminate superoxide-producing mitochondria and cell. FEBS Lett 397: 7-10, 1996.

41. Zamzami N, Susin SA, Marchetti P,Hirsch T, Gómez-Monterrey I, Castedo M and Kroemer G: Mitochondrial control of nuclear apoptosis. J Exp Med 183: 1533-1544, 1996.

42. Xiong Y, Ye T, Wang M, Xia Y, Wang N, Song X, Wang F, Liu L, Zhu Y, Yang F, et al: A novel cinnamide YLT26 induces breast cancer cells apoptosis via ROS-mitochondrial apoptotic pathway in vitro and inhibits lung metastasis in vivo. Cell Physiol Biochem 34: 1863-1876, 2014.

43. Finkel T: Signal transduction by reactive oxygen species. J Cell Biol 194: 7-15, 2011

44. Zhang T, Li J, Dong Y, Zhai D, Lai L, Dai F, Deng H, Chen Y, Liu M and Yi Z: Cucurbitacin E inhibits breast tumor metastasis by suppressing cell migration and invasion. Breast Cancer Res Treat 135: 445-458, 2012.

45. Thiery JP, Acloque H, Huang RY and Nieto MA: Epithelial-mesenchymal transitions in development and disease. Cell 139: 871-890, 2009.

46. Bhuvarahamurthy V, Kristiansen GO, Johannsen M, Loening SA, Schnorr D, Jung K and Staack A: In situ gene expression and localization of metalloproteinases MMP1, MMP2, MMP3, MMP9, and their inhibitors TIMP1 and TIMP2 in human renal cell carcinoma. Oncol Rep 15: 1379-1384, 2006.

47. Kim SY, Yi HK, Yun BS, Lee DY, Hwang PH, Park HR and Kim MS: The extract of the immature fruit of Poncirus trifoliato induces apoptosis in colorectal cancer cells via mitochondrial autophagy. Food Sci Hum Wellness, 2020.

48. Braillon A: Hepatocellular carcinoma. Lancet 380: 469-471, 2012.

49. Budhu A, Forgues M, Ye QH, Jia HL, He P, Zanetti KA, Kammula US, Chen Y, Qin LX, Tang ZY and Wang XW: Prediction of venous metastases, recurrence, and prognosis in hepatocellular carcinoma based on a unique immune response signature of the liver microenvironment. Cancer Cell 10: 99-111, 2006

50. Min SH, Zhou XZ and Lu KP: The role of Pin1 in the development and treatment of cancer. Arch Pharm Res 39: 1609-1620, 2016.

51. Wulf GM, Ryo A, Wulf GG, Lee SW, Niu T, Petkova V and Lu KP: Pin 1 is overexpressed in breast cancer and cooperates with Ras signaling in increasing the transcriptional activity of c-Jun towards cyclin D1. EMBO J 20: 3459-3472, 2001.

52. Xu GG and Etzkorn FA: Pin1 as an anticancer drug target. Drug News Perspect 22: 399-407, 2009.

53. Nakayama K, Hatakeyama S, Maruyama S, Kikuchi A, Onoé K, Good RA and Nakayama KI: Impaired degradation of inhibitory subunit of NF-kappa B (I kappa B) and beta-catenin as a result of targeted disruption of the beta-TrCP1 gene. Proc Natl Acad Sci USA 100: 8752-8757, 2003.

54. Cho YS, Park SY, Kim DJ, Lee SH, Woo KM, Lee KA, Lee YJ, Cho YY and Shim JH: TPA-induced cell transformation provokes a complex formation between Pin 1 and $90 \mathrm{kDa}$ ribosomal protein S6 kinase 2. Mol Cell Biochem 367: 85-92, 2012.

55. Nejak-Bowen KN, Thompson MD, Singh S, Bowen WC Jr, Dar MJ, Khillan J, Dai C and Monga SP: Accelerated liver regeneration and hepatocarcinogenesis in mice overexpressing serine-45 mutant beta-catenin. Hepatology 51: 1603-1613, 2010.

56. Cheng CW, Chow AK, Pang R, Fok EW, Kwong YL and Tse E: PIN1 inhibits apoptosis in hepatocellular carcinoma through modulation of the antiapoptotic function of survivin. Am J Pathol 182: 765-775, 2013

57. Brembeck FH, Rosário M and Birchmeier W: Balancing cell adhesion and Wnt signaling, the key role of beta-catenin. Curr Opin Genet Dev 16: 51-59, 2006.

58. Inagawa $\mathrm{S}$, Itabashi $\mathrm{M}$, Adachi S, Kawamoto $\mathrm{T}$, Hori $\mathrm{M}$, Shimazaki J, Yoshimi F and Fukao K: Expression and prognostic roles of beta-catenin in hepatocellular carcinoma: Correlation with tumor progression and postoperative survival. Clin Cancer Res 8: 450-456, 2002.

59. David CJ and Manley JL: Alternative pre-mRNA splicing regulation in cancer: Pathways and programs unhinged. Genes Dev 24: 2343-2364, 2010 
60. Ko H, Kim S, Jin CH, Lee E, Ham S, Yook JI and Kim K: Protein kinase casein kinase 2-mediated upregulation of $\mathrm{N}$-cadherin confers anoikis resistance on esophageal carcinoma cells. Mol Cancer Res 10: 1032-1038, 2012.

61. Hanahan D and Weinberg RA: Hallmarks of cancer: The next generation. Cell 144: 646-674, 2011.

62. Shenoy AK, Jin Y, Luo H, Tang M, Pampo C, Shao R, Siemann DW, Wu L, Heldermon CD, Law BK, et al: Epithelial-to-mesenchymal transition confers pericyte properties on cancer cells. J Clin Invest 126: 4174-4186, 2016.

63. Tsai JH and Yang J: Epithelial-mesenchymal plasticity in carcinoma metastasis. Genes Dev 27: 2192-2206, 2013.

64. Cheng Y, Chen T, Yang X, Xue J and Chen J: Atractylon induces apoptosis and suppresses metastasis in hepatic cancer cells and inhibits growth in vivo. Cancer Manag Res 11: 5883-5894, 2019

65. Kamat AA, Fletcher M, Gruman LM, Mueller P, Lopez A, Landen CN Jr, Han L, Gershenson DM and Sood AK: The clinical relevance of stromal matrix metalloproteinase expression in ovarian cancer. Clin Cancer Res 12: 1707-1714, 2006.

66. Deryugina EI and Quigley JP: Tumor angiogenesis: MMP-mediated induction of intravasation- and metastasissustaining neovasculature. Matrix Biol 44-46: 94-112, 2015.

67. Iyer RP, Patterson NL, Fields GB and Lindsey ML: The history of matrix metalloproteinases: Milestones, myths, and misperceptions. Am J Physiol Heart Circ Physiol 303: H919-H930, 2012.

68. Kessenbrock K, Wang CY and Werb Z: Matrix metalloproteinases in stem cell regulation and cancer. Matrix Biol 44-46: 184-190, 2015.

69. Zhu M, Li W, Dong X, Chen Y, Lu Y, Lin B, Guo J and Li M: Benzyl-isothiocyanate induces apoptosis and inhibits migration and invasion of hepatocellular carcinoma cells in vitro. J Cancer 8: 240-248, 2017.
70. Kang $\mathrm{MH}$ and Reynolds CP: Bcl-2 inhibitors: Targeting mitochondrial apoptotic pathways in cancer therapy. Clin Cancer Res 15: 1126-1132, 2009.

71. Wang F, He L, Dai WQ, Xu YP, Wu D, Lin CL, Wu SM, Cheng P, Zhang Y, Shen M, et al: Salinomycin inhibits proliferation and induces apoptosis of human hepatocellular carcinoma cells in vitro and in vivo. PLoS One 7: e50638, 2012.

72. Zhang Z, Lapolla SM, Annis MG, Truscott M, Roberts GJ, Miao Y, Shao Y, Tan C, Peng J, Johnson AE, et al: Bcl-2 homodimerization involves two distinct binding surfaces, a topographic arrangement that provides an effective mechanism for Bcl-2 to capture activated Bax. J Biol Chem 279: 43920-43928, 2004.

73. Dewson G and Kluck RM: Mechanisms by which Bak and Bax permeabilise mitochondria during apoptosis. J Cell Sci 122: 2801-2808, 2009.

74. Indran IR, Tufo G, Pervaiz S and Brenner C: Recent advances in apoptosis, mitochondria and drug resistance in cancer cells. Biochim Biophys Acta 1807: 735-745, 2011.

75. Ly JD, Grubb DR and Lawen A: The mitochondrial membrane potential (deltapsi(m)) in apoptosis; an update. Apoptosis 8: $115-128,2003$

76. Ye T, Zhu S, Zhu Y, Feng Q, He B, Xiong Y, Zhao L, Zhang Y, Yu L and Yang L: Cryptotanshinone induces melanoma cancer cells apoptosis via ROS-mitochondrial apoptotic pathway and impairs cell migration and invasion. Biomed Pharmacother 82: 319-326, 2016.

This work is licensed under a Creative Commons Attribution-NonCommercial-NoDerivatives 4.0 International (CC BY-NC-ND 4.0) License. 